\title{
Compressive Strength Prediction of Stabilized Dredged Sediments Using Artificial Neural Network
}

\author{
Van Quan Tran (iD \\ University of Transport Technology, Hanoi 100000, Vietnam \\ Correspondence should be addressed to Van Quan Tran; quantv@utt.edu.vn
}

Received 11 November 2020; Revised 20 February 2021; Accepted 13 March 2021; Published 22 March 2021

Academic Editor: Wei Liu

Copyright (c) 2021 Van Quan Tran. This is an open access article distributed under the Creative Commons Attribution License, which permits unrestricted use, distribution, and reproduction in any medium, provided the original work is properly cited.

\begin{abstract}
Stabilized dredged sediments are used as a backfilling material to reduce construction costs and a solution to environmental protection. Therefore, the compressive strength is an important criterion to determine the stabilized dredged sediments application such as road construction, building construction, and highway construction. Using the traditional method such as empirical approach and experimental methods, the determination of compressive strength of stabilized dredged sediments is difficult due to the complexity of this composite material. In this investigation, the artificial neural network (ANN) model is introduced to forecast the compressive strength. To perform the simulation, 51 experimental datasets were collected from the literature. The dataset consists of 4 input variables (water content, cement content, air foam content, and waste fishing net content) and output variable (compressive strength). Evaluation of the models was made and compared on training dataset ( $70 \%$ data) and testing dataset (30\% remaining data) by the criteria of Pearson's correlation coefficient (R), Mean Absolute Error (MAE), and Root Mean Square Error (RMSE). The results show that the ANN model can accurately predict the compressive strength of stabilized dredged sediments with low water content. The cement content is the most important input affecting the unconfined compressive strength. The important input affecting the unconfined compressive strength can be in the following order: cement content $>$ air foam content $>$ water content $>$ waste fishing net.
\end{abstract}

\section{Introduction}

Vietnam has a coastline about $3260 \mathrm{~km}$ followed by 49 large and small harbors with a system of estuarine serving ships. Especially in the Mekong Delta, transportation on the river system plays an important role in regional economic development. The dredged sludge needs to be treated to avoid environmental pollution. Furthermore, the dredged sludge amount is a challenge posed in the exploitation of estuaries and harbors. The Mekong Delta is characterized by soft soils, so that the requirement of soft soils improvement for construction works is very large. Stabilization/solidification is widely used in the treatment of contaminated sediments by mixing binders materials into the dredged sediments. Using stabilization/solidification technology has two purposes, which are (i) treating the environment and (ii) using a backfilling material to reduce construction costs and use of recycled materials such as dredged sediments, respectively.

Stabilization/solidification is the improvement of the physical dredged sediments properties such as compressive strength, liquid limit, plastic limit, viscosity, and permeability [1-5]. Some binders are commonly used such as Portland cement, lime, limestone, fly ash, slag, gypsum, phosphorus, and many other commercial products. Considered as a construction material, Tsuchida [6] used dredged sediments, cement, air foam, and waste fishing net to form a lightweight material (cf. Figure 1). Dredged sediment has high water content; light foam nature reduces the density of stabilized dredged sediment but increases porosity. The waste fishing net has high shear strength. Therefore, the compressive strength of stabilized dredged sediments is importantly affected by the mix design (cement, water, air foam, and waste fishing net). That implies to be difficult to determine empirically relationship between the compressive strength and the composition of mix design, so that, in order to formulate the complex relationship, a suitable prediction model is demanded.

Over the past four decades, the method of artificial intelligence (AI) based on computer science has received a 


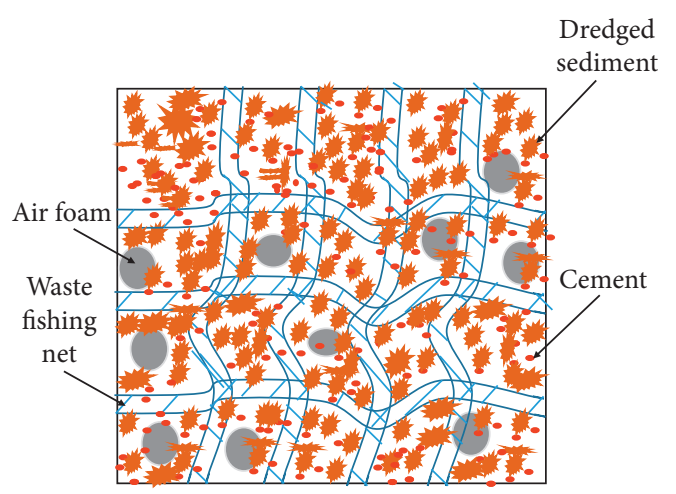

Figure 1: Mix design of stabilized dredged sediments using cement, air foam, and waste fishing net according to [7].

lot of attention from scientists applying on abundant sector such as earth science $[8,9]$ and civil engineering $[10,11]$. Among AI algorithms, artificial neural networks (ANN) are often applied to solve various technical problems. Many complex issues related to structural engineering [12] and materials science [11, 13-17] have been successfully solved. Therefore, in this article, the authors propose the application of ANN model to predict compressive strength of stabilized dredged sediments using mix design, cement, air foam, and waste fishing net.

\section{Machine Learning Method}

2.1. Artificial Neural Network. The artificial neural network (ANN) is a mathematical model designed to perform a specific task, based on processing information of human brain with neurons process. Until now, ANN has been successfully used in many areas of life [18]. Regarding functional approximations, ANN model solutions are often more accurate than those provided by traditional methods, such as multivariate nonlinear regressions. The ANN structure is created by three or more layers including an input layer, an output layer, and one or more hidden layers (Figure 2). The input layer takes the values of the input and sends them to the available neurons in the hidden layer. Within each neuron, a weighted input is calculated. The sum of this value and the deviation value is modified by the activation function. Finally, the output signal is sent to the neurons in the next layer.

The mathematical process can be constructed as follows:

$$
y_{j}=f\left(\sum_{i=1}^{n} w_{i j} x_{i}+b_{j}\right),
$$

where $x_{i}$ is input value $I, y_{j}$ is output value $I$, and $w_{i j}$ and $b_{j}$ are weight and deviation value.

A tangent hyperbolic function was used in this investigation, as it could lead to more accurate results. That is confirmed by the investigation of Karlik and Olgac [19]. This function varies from -1 to 1 and is expressed as follows:

$$
y_{i}=f(\text { net })=\frac{2}{1+e^{-2 . n e t}}-1,
$$

where $f$ is the activation function according to the terms of the calculated network value.

Neural networks need training to show effective performance. Training means that the weight and deviation of network are determined such that the minimum error between the target (actual value) and the output (network value) occurs. Therefore, during the training of neural networks, back-propagation algorithms (BP) are often used to train the network. The Levenberg-Marquardt algorithm (LMA) is usually the fastest back-propagation algorithm for tool training $[20,21]$. Therefore, LMA is applied in this study.

The LM algorithm provides a solution called least squares of the following form:

$$
f(x)=\frac{1}{2} \sum_{j=1}^{m} r_{j}^{2}(x),
$$

where $x=\left(x_{1}, x_{2}, \ldots, x_{n}\right)$ is vector and $r_{j}$ is function of $\mathfrak{R}^{n} \longrightarrow \mathfrak{R}$. $r_{j}$ is $r$ when $m \geq n$. For simplicity, $f$ is represented as a residual vector $r: \mathfrak{R}^{n} \longrightarrow \mathfrak{R}^{m}$ and is shown as follows:

$$
r(x)=\left(r_{1}(x), r_{2}(x), \ldots, r_{m}(x)\right) .
$$

At this time, $f$ can be rewritten as $f(x)=(1 / 2)\|r(x)\|^{2}$. The derivative of $f$ can be written in the Jacobi matrix and is defined as follows:

$$
J(x)=\frac{\partial r_{j}}{\partial x_{i}} ; \quad 1 \leq j \leq m ; 1 \leq i \leq n .
$$

First, consider that every function $r_{i}$ is linear. Here, Jacobian is constant and therefore is given by the square root as follows:

$$
f(x)=\frac{1}{2}\|J x+r(0)\|^{2} .
$$

We get

$$
\begin{aligned}
\nabla f(x) & =J^{T}(J x+r), \\
\nabla^{2} f(x) & =J^{T} J .
\end{aligned}
$$

Placing $\nabla f(x)=0$, we get $x_{\min }=-\left(J^{T} J\right)^{-1} J^{T} r$. This is the result of the normal equation. Going back $\mathrm{xxxx}^{\text {"the }}$ nonlinear case"

$$
\begin{gathered}
\nabla f(x)=\sum_{j=1}^{m} r_{j}(x) \nabla r_{j}(x)=J(x)^{T} r(x), \\
\nabla^{2} f(x)=J(x)^{T} J(x)+\sum_{j=1}^{m} r_{j}(x) \nabla^{2} r_{j}(x) .
\end{gathered}
$$

The special property of square problems is with the Jacobi matrix; it is possible to get the Hessian matrix basically $\left(\nabla^{2} f(x)\right)$ if possible approximate $r_{j s}$ by linear functions $\nabla^{2} r_{j}(x)$ is small or small residual $\left(r_{j}(x)\right)$. The Hessian matrix in this case simply becomes

$$
\nabla^{2} f(x)=J(x)^{T} J(x) .
$$

The results are the same as those for linear cases. 


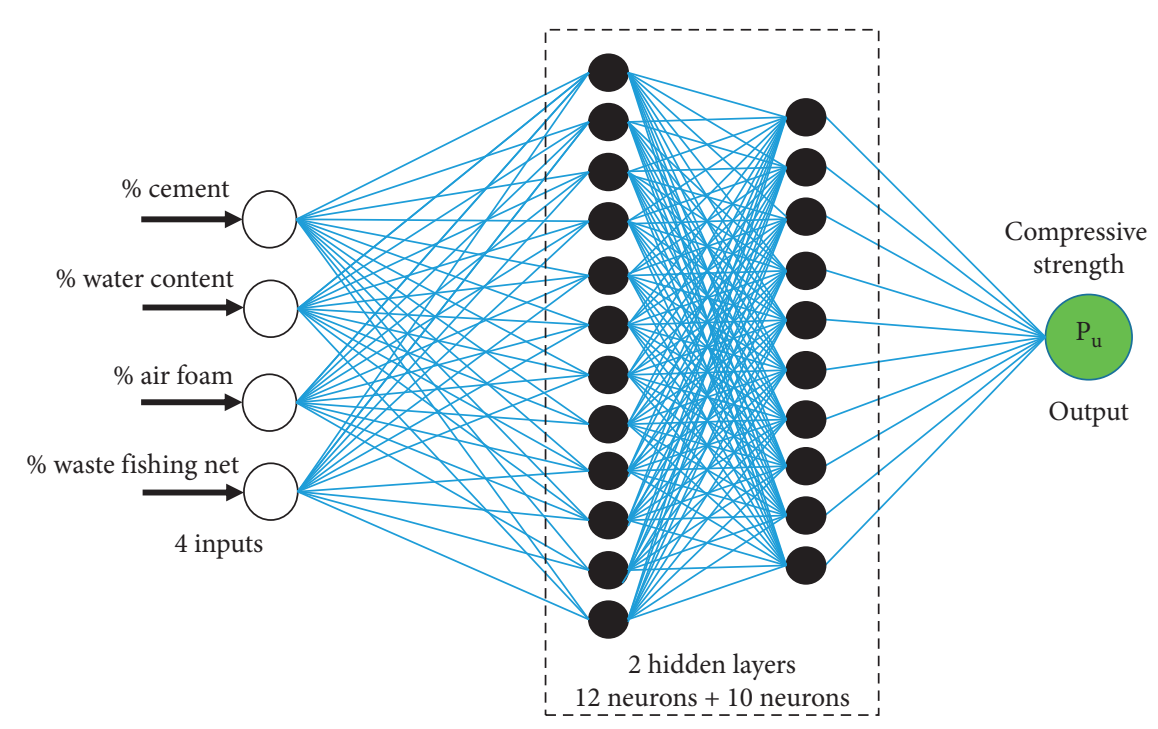

Figure 2: Architecture for the developed ANN: 4 inputs, 2 hidden layers, and 1 output.

2.2. Structure of the ANN Model. The effectiveness of the ANN model depends on the structure of the neural network $(\mathrm{NN})$, that is, the number of hidden layers and the number of neurons. The ANN structure was chosen to predict the stabilized dredged sediments in this investigation, including 4 layers. The input layer consists of 4 neurons corresponding to 4 input variables (cement content, water content, air foam content, and waste fishing net content), and the output layer includes 1 neuron representing compressive strength. According to $[22,23]$, the accuracy of the ANN model is depends strongly on the structure of the ANN model such as number of hidden layers and number of neurons in each hidden layer. For comparison with the ANN model proposed by Park and Kim [7], number of hidden layers is assumed to be equal to 2 and number of neurons in each hidden layer is manually chosen. The number of neurons in each hidden layer is varied from 9 to 12 neurons to cover the range of neuron as suggested in previously research, such as in the works of Neville [24] and Hush [25]. Therefore, 16 ANN architectures are built. The performance of each model is evaluated to determine the best ANN architecture.

2.3. Performance Evaluation. In this investigation, the three criteria used are correlation coefficients (R) (Pearson's correlation coefficient), Mean Absolute Error (MAE), and Root Mean Square Error (RMSE) to evaluate the accuracy of the developed ANN model [26]:

$$
\begin{aligned}
\text { RMSE } & =\sqrt{\frac{1}{N} \sum_{j=1}^{N}\left(p_{0, j}-p_{t, j}\right)^{2}}, \\
R & =\frac{\sum_{j=1}^{N}\left(p_{0, j}-\bar{p}_{0}\right)\left(p_{t, j}-\bar{p}_{t}\right)}{\sqrt{\sum_{j=1}^{N}\left(p_{0, j}-\bar{p}_{0}\right)^{2} \sum_{j=1}^{N}\left(p_{t, j}-\bar{p}_{t}\right)^{2}}} \\
\operatorname{MAE} & =\frac{1}{N} \sum_{j=1}^{N}\left(p_{0, j}-p_{t, j}\right),
\end{aligned}
$$

where $N$ is the number of datasets, $p_{0}$ and $\bar{p}_{0}$ are the actual experiment value and the average experimental value, and $p_{\mathrm{t}}$ and $\bar{p}_{t}$ are the predicted value and the average predicted value, calculated according to the ANN model. $R$ measures the predicted and experimental value association; if $R$ is closer to 1 , the ANN model is more accurate. RMSE calculates the square root average difference between the expected values and the experimental values and the difference between the experimental and the predicted values is determined by MAE criteria.

\section{Database Construction}

In this investigation, the data was extracted from [7], in which 51 experimental pieces of data of stabilized dredged sediments are designed with cement, air foam, and waste fishing net. The ANN model uses 4 input variables: (1) cement content (\% by weight), (2) water content, (3) air foam content ( $\%$ by weight), and (4) waste fishing net content ( $\%$ by weight). Output parameter is considered to be unconfined compressive strength $P_{\mathrm{u}}\left(\mathrm{kN} / \mathrm{m}^{2}\right)$. The dataset was randomly divided into two subdatasets, and $70 \%$ of the data were used to train the ANN models corresponding to 36 samples. The remaining $30 \%$ of the data correspond to the 15 samples used in the testing model. The initial statistical analysis of the dataset is presented in Table 1.

The used cement content ranges from $8 \%$ to $20 \%$ by weight of untreated sediment (mean value of $12.5 \%$ and standard deviation of $2.5 \%$ ). The water content ranges from $125 \%$ to $250 \%$ by weight of untreated sediment (mean value of $168.2 \%$ and standard deviation of $32.5 \%$ ). The used air foam ranges from $1 \%$ to $5 \%$ by weight of untreated sediment (mean value of $2.4 \%$ and standard deviation of $1 \%$ ). The used waste fishing net ranges from $0 \%$ to $0.2 \%$ by weight of untreated sediment (mean value of $0.1 \%$ and standard deviation of $0.1 \%)$. 
TABLe 1: Initial statistical analysis of the dataset.

\begin{tabular}{|c|c|c|c|c|c|}
\hline Variable & Cement & Water content & Air foam & Waste fishing net & $P_{\mathrm{u}}$ \\
\hline Unit & $\%$ by weight & $\%$ by weight & $\%$ by weight & \% by weight & $\mathrm{kN} / \mathrm{m}^{2}$ \\
\hline Role & Input & Input & Input & Input & Output \\
\hline Count & 51.0 & 51.0 & 51.0 & 51.0 & 51.0 \\
\hline Mean & 12.5 & 168.2 & 2.4 & 0.1 & 38.0 \\
\hline $\operatorname{Std}^{\mathrm{a}}$ & 2.5 & 32.5 & 1.0 & 0.1 & 26.5 \\
\hline Min & 8.0 & 125.0 & 1.0 & 0.0 & 7.9 \\
\hline$Q_{25}$ & 12.0 & 156.0 & 2.0 & 0.0 & 15.7 \\
\hline$Q_{50}$ & 12.0 & 156.0 & 2.0 & 0.1 & 27.2 \\
\hline$Q_{75}$ & 12.0 & 171.5 & 2.0 & 0.1 & 60.4 \\
\hline Max & 20.0 & 250.0 & 5.0 & 0.2 & 100.7 \\
\hline
\end{tabular}

${ }^{\mathrm{a}}$ Standard deviation.

\section{Results and Discussion}

During back-propagation network training, the cycle of sending all training samples across the network is called an epoch. The training process will be repeated until the error at the network output reaches an acceptable value (less than the initial specified error threshold). The objective of this process is to minimize the error between actual data and simulation data. Training and testing processes are also used to determine the optimal number of epochs for the model. Figure 3 shows the best performance of the ANN models for training and test processes with 500 epochs. This number of epochs has been chosen to prevent the proposed ANN model from overfitting.

With ANN architecture containing 2 hidden layers, 16 structures are investigated, as shown in Figure 4 for the training datasets. Figures 4(a)-4(c) show the values of $R$, RMSE, and MAE, respectively. It is worth nothing that 10 neurons in the second hidden layer produce higher prediction accuracy. The best architecture containing, respectively, 12 neurons and 10 neurons for first and second hidden layers is observed to achieve the best accuracy of training part with highest value of $R$ and lowest values of RMSE and MAE. That is a good example to show that a suitable ANN architecture should be determined before performing any further simulations. The best ANN architecture is used for predicting the compressive strength of stabilized dredged sediments.

Using the ANN model [4-12], the prediction of stabilized dredged sediments compressive strength for training and testing part is shown in Figures 5(a) and 5(b), respectively. Figure 5 shows that the ANN model's results and experimental results are almost identical for each sample in the training phase. It shows that the prediction capacity of the ANN model is excellent. Therefore, the ANN model can predict relatively accurately for the testing phase.

The regression model for the training and testing parts is shown in Figures 6(a) and 6(b), respectively. From the figure above, we can see that the prediction ability of the ANN model is quite close to the experimental compressive strength, but there are still high errors for testing part, high water content such as $218 \%$ (see Table 2).

The correlation value obtained for training is $R=0.95$ and the control is $R=0.94$. This shows that applying the

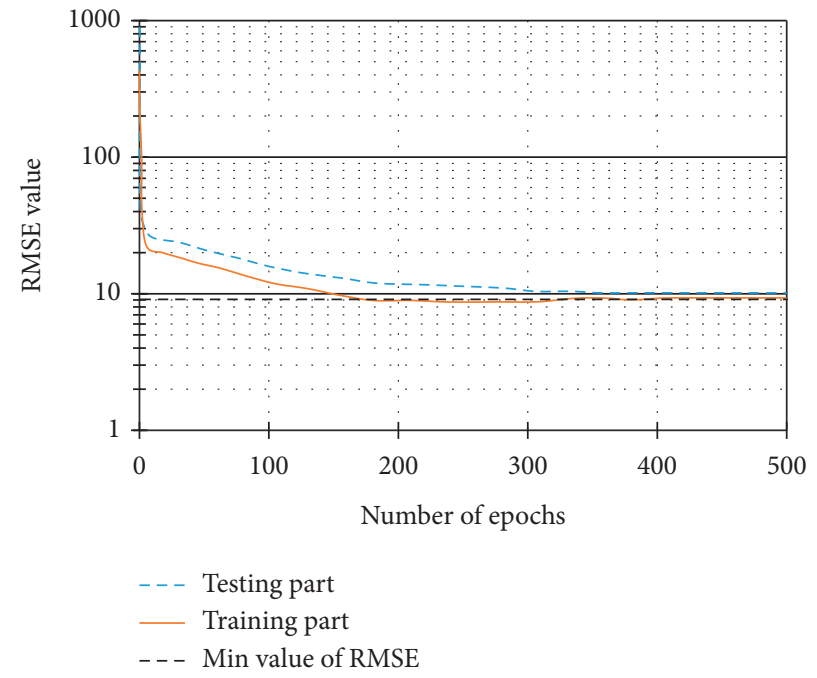

FIGURE 3: RMSE value of training part and testing part in function of number of epochs.

ANN model for predicting the compressive strength of stabilized dredged sediments is very feasible due to high accuracy and low error. For training dataset, the function " $y=x-0.15$ " is set to show the correlation between experimental data and the ANN model's data. Similarly, the function " $y=1.1 x+0.17$ " is set for correlation in the control dataset. For RMSE indicators, the biggest errors are 9.1948 and 10.3390 with training and testing, respectively. For MAE indicators, the biggest errors are 3.9001 and 8.6535, respectively, for training and testing (Table 3 ). Therefore, the ability to predict is relatively high.

To prove the accuracy of the ANN model, Table 2 shows the 51 experimental pieces of data of [7] and compares them with the ANN model's results. It can be seen that the ANN simulation's results give very low errors in most cases, except for the high water content. The biggest error of the ANN model compared to the experiment is $382.2 \%$ and the lowest is $0.0 \%$ for $156 \%$ of water content. The ANN model predicts relatively correctly with lower water content, which importantly affects the accuracy of the ANN model.

In fact, the investigation of Park and Kim [7] has developed an ANN model for predicting the unconfined compressive strength of reinforced lightweight soil. The 


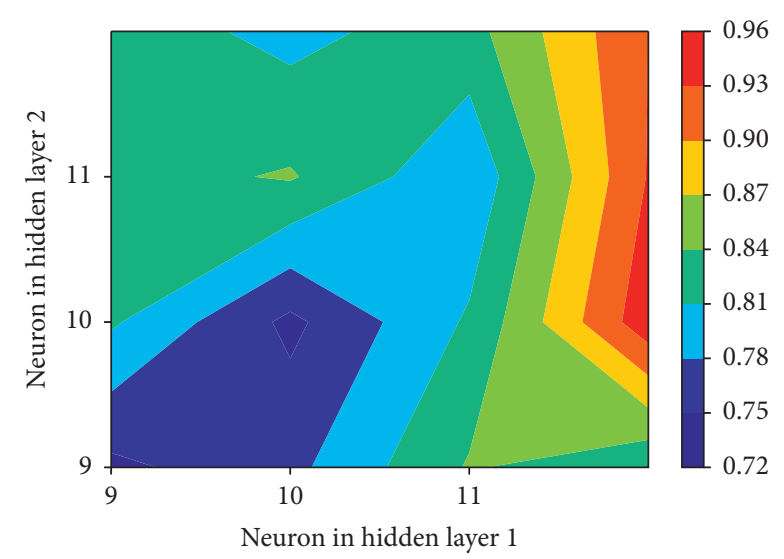

(a)

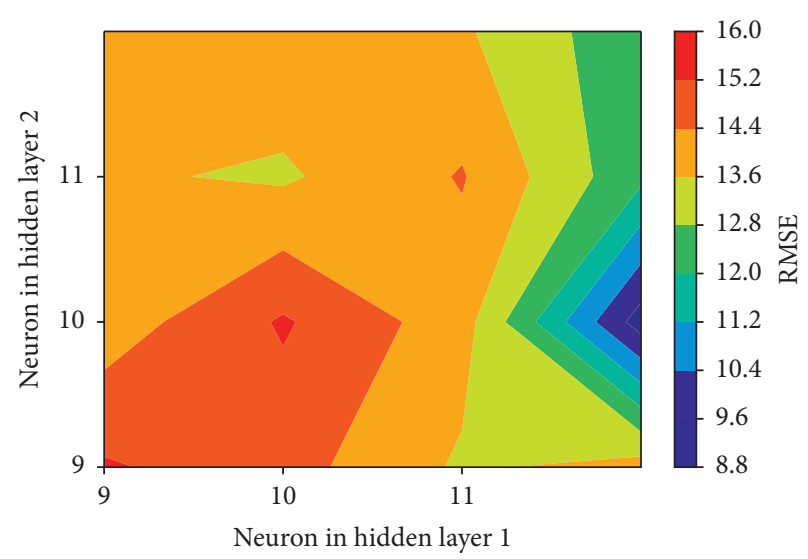

(b)

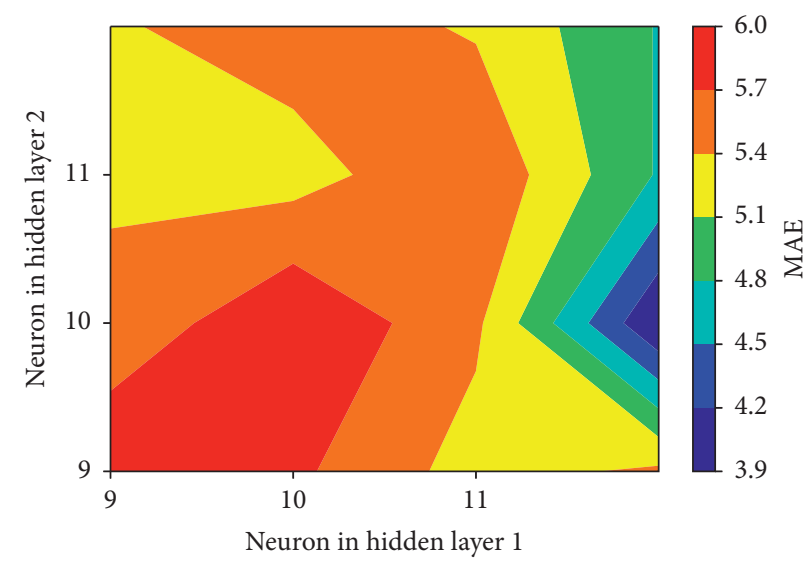

(c)

FIGURE 4: Color map of ANN with 2 hidden layers in function of the neuron in the hidden layer for the training part with respect to (a) values of $R$, (b) value of RMSE, and (c) value of MAE.

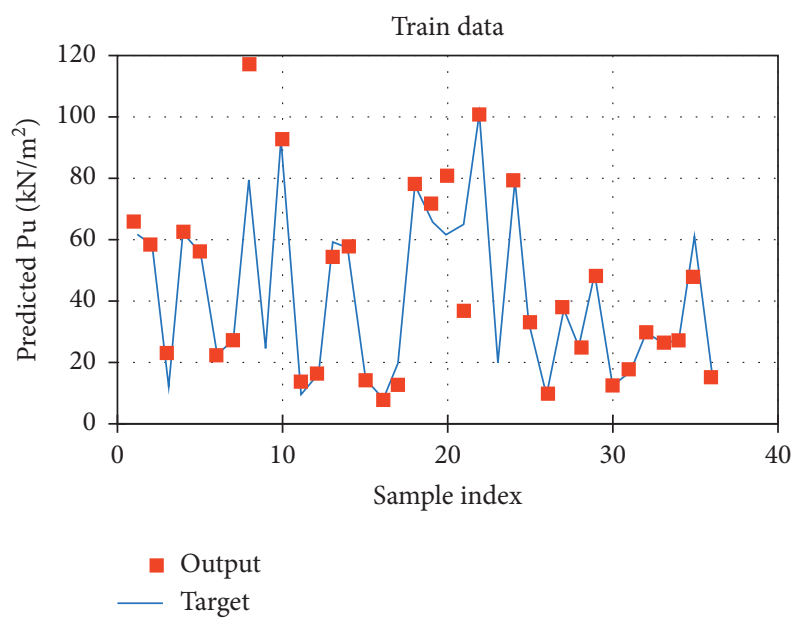

(a)

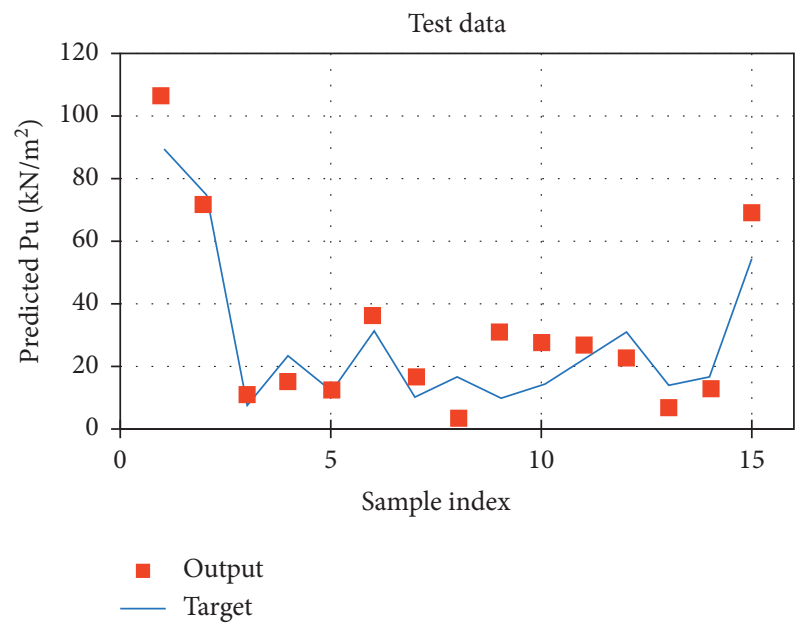

(b)

FIgUre 5: Predicted compressive strength of stabilized dredged sediments by the ANN model. (a) Training. (b) Testing.

ANN structure in this investigation is a single hidden layer containing 4 neurons. The performance of this ANN model was only evaluated through Pearson's correlation coefficient $R$, which was equal to 0.97 and 0.96 for training and testing parts, respectively. The number of hidden layers such as single layer or multiple hidden layers is always a big challenge of the ANN structure $[22,23]$. In our investigation, a new ANN structure is developed consisting of two hidden 


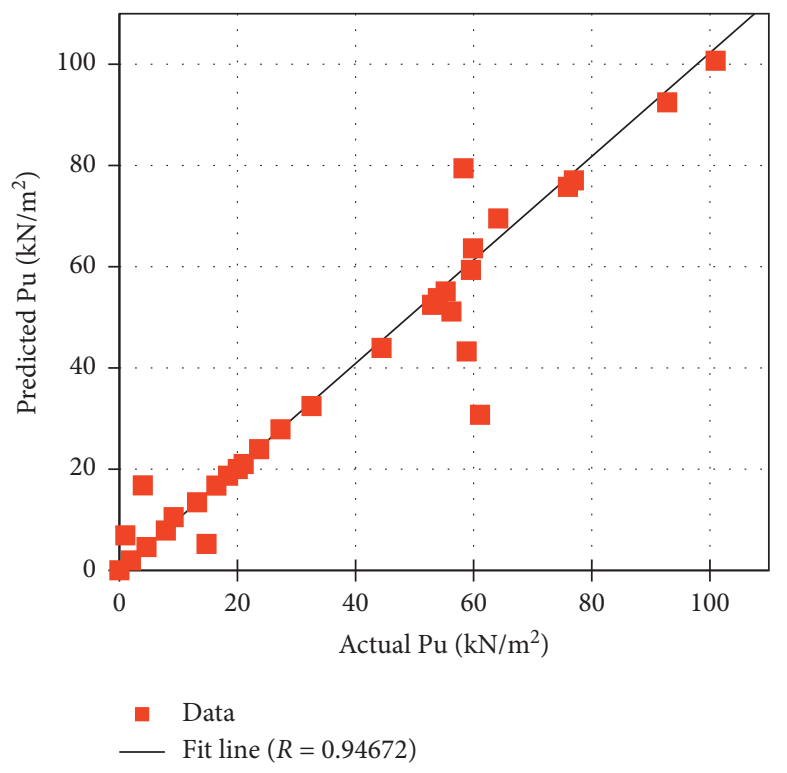

(a)

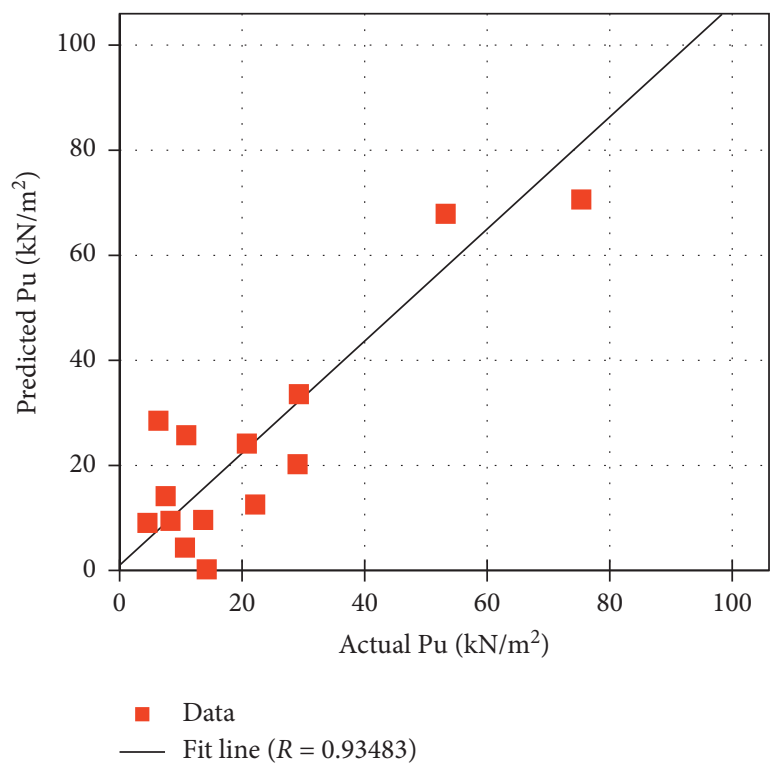

(b)

FIgURE 6: ANN regression results for (a) training and (b) testing.

TABLE 2: Comparison of experimental results and ANN model results with error (ANN-EXP)/EXP.

\begin{tabular}{|c|c|c|c|c|c|c|c|c|}
\hline Sample & Dataset & \% cement & $\%$ water & $\%$ air foam & $\%$ waste & Experimental & ANN & Error (\%) \\
\hline 01 & Training & 12 & 156 & 1 & 0.143 & 62.81 & 66.49 & 5.54 \\
\hline 02 & Training & 12 & 156 & 1 & 0.179 & 58.9 & 58.90 & 0.00 \\
\hline 03 & Training & 12 & 156 & 5 & 0 & 11.56 & 23.63 & 51.08 \\
\hline 04 & Training & 12 & 125 & 2 & 0.036 & 63.02 & 63.02 & 0.00 \\
\hline 05 & Training & 12 & 156 & 2 & 0.143 & 56.22 & 56.22 & 0.00 \\
\hline 06 & Training & 12 & 156 & 4 & 0.036 & 23.09 & 23.09 & 0.00 \\
\hline 07 & Training & 12 & 156 & 2 & 0.179 & 27.23 & 27.23 & 0.00 \\
\hline 08 & Training & 20 & 156 & 2 & 0 & 80.16 & 116.96 & 31.46 \\
\hline 09 & Training & 12 & 156 & 3 & 0.179 & 25.2 & 25.20 & 0.00 \\
\hline 10 & Training & 16 & 156 & 2 & 0.036 & 93.14 & 93.14 & 0.00 \\
\hline 11 & Training & 12 & 250 & 2 & 0.036 & 8.7 & 14.50 & 40.00 \\
\hline 12 & Training & 12 & 218 & 2 & 0 & 16.24 & 16.24 & 0.00 \\
\hline 13 & Training & 12 & 156 & 2 & 0.107 & 59.49 & 55.21 & -7.75 \\
\hline 14 & Training & 12 & 156 & 1 & 0 & 57.56 & 57.56 & 0.00 \\
\hline 15 & Training & 12 & 218 & 2 & 0.179 & 14.94 & 14.94 & 0.00 \\
\hline 16 & Training & 12 & 250 & 2 & 0.107 & 7.93 & 7.93 & 0.00 \\
\hline 17 & Training & 12 & 218 & 2 & 0.036 & 21.44 & 12.97 & -65.27 \\
\hline 18 & Training & 16 & 156 & 2 & 0.107 & 78.19 & 78.19 & 0.00 \\
\hline 19 & Training & 12 & 156 & 1 & 0.107 & 66.79 & 72.41 & 7.76 \\
\hline 20 & Training & 12 & 125 & 2 & 0.107 & 61.28 & 81.51 & 24.82 \\
\hline 21 & Training & 16 & 156 & 2 & 0.179 & 64.37 & 36.55 & -76.10 \\
\hline 22 & Training & 20 & 156 & 2 & 0.143 & 100.7 & 100.70 & 0.00 \\
\hline 23 & Training & 8 & 156 & 2 & 0.143 & 20.1 & 20.10 & 0.00 \\
\hline 24 & Training & 12 & 125 & 2 & 0.143 & 79 & 79.00 & 0.00 \\
\hline 25 & Training & 12 & 156 & 2 & 0 & 33.15 & 33.15 & 0.00 \\
\hline 26 & Training & 8 & 156 & 2 & 0.179 & 9.76 & 9.76 & 0.00 \\
\hline 27 & Training & 12 & 125 & 2 & 0 & 37.95 & 37.95 & 0.00 \\
\hline 28 & Training & 12 & 156 & 3 & 0 & 25.06 & 25.06 & 0.00 \\
\hline 29 & Training & 12 & 125 & 2 & 0.179 & 48.69 & 48.69 & 0.00 \\
\hline 30 & Training & 12 & 250 & 2 & 0 & 12.2 & 12.20 & 0.00 \\
\hline 31 & Training & 8 & 156 & 2 & 0.036 & 16.21 & 18.13 & 10.58 \\
\hline 32 & Training & 12 & 187 & 2 & 0.036 & 29.76 & 29.76 & 0.00 \\
\hline 33 & Training & 12 & 187 & 2 & 0.107 & 26.35 & 26.35 & 0.00 \\
\hline 34 & Training & 12 & 156 & 3 & 0.107 & 27.4 & 27.40 & 0.00 \\
\hline
\end{tabular}


TABLE 2: Continued.

\begin{tabular}{|c|c|c|c|c|c|c|c|c|}
\hline Sample & Dataset & $\%$ cement & $\%$ water & $\%$ air foam & $\%$ waste & Experimental & ANN & Error (\%) \\
\hline 35 & Training & 12 & 156 & 2 & 0.036 & 61.86 & 48.14 & -28.51 \\
\hline 36 & Training & 12 & 156 & 5 & 0.143 & 15.2 & 15.20 & 0.00 \\
\hline 37 & Testing & 20 & 156 & 2 & 0.107 & 89.82 & 106.17 & 15.40 \\
\hline 38 & Testing & 16 & 156 & 2 & 0.143 & 76.01 & 71.65 & -6.08 \\
\hline 39 & Testing & 12 & 250 & 2 & 0.143 & 8.17 & 11.94 & 31.57 \\
\hline 40 & Testing & 12 & 156 & 4 & 0.107 & 25.06 & 15.35 & -63.29 \\
\hline 41 & Testing & 12 & 156 & 5 & 0.179 & 11.71 & 12.52 & 6.46 \\
\hline 42 & Testing & 12 & 187 & 2 & 0.143 & 32.06 & 36.16 & 11.34 \\
\hline 43 & Testing & 8 & 156 & 2 & 0 & 10.86 & 17.17 & 36.76 \\
\hline 44 & Testing & 12 & 218 & 2 & 0.143 & 17.50 & 3.63 & -382.2 \\
\hline 45 & Testing & 12 & 187 & 2 & 0 & 10.15 & 30.94 & 67.20 \\
\hline 46 & Testing & 12 & 156 & 4 & 0.179 & 14.42 & 28.16 & 48.79 \\
\hline 47 & Testing & 12 & 156 & 3 & 0.036 & 23.85 & 26.76 & 10.87 \\
\hline 48 & Testing & 12 & 156 & 3 & 0.143 & 31.70 & 23.20 & -36.66 \\
\hline 49 & Testing & 12 & 187 & 2 & 0.179 & 13.95 & 7.33 & -90.24 \\
\hline 50 & Testing & 12 & 156 & 5 & 0.036 & 16.81 & 12.93 & -30.03 \\
\hline 51 & Testing & 12 & 156 & 1 & 0.036 & 54.87 & 68.95 & 20.43 \\
\hline
\end{tabular}

TABle 3: Pearson's correlation coefficient (R), Mean Absolute Error (MAE), and Root Mean Square Error (RMSE).

\begin{tabular}{lcc}
\hline & Training & Testing \\
\hline RMSE & 9.1948 & 10.3390 \\
MAE & 3.9001 & 8.6535 \\
R & 0.95 & 0.94 \\
\hline
\end{tabular}

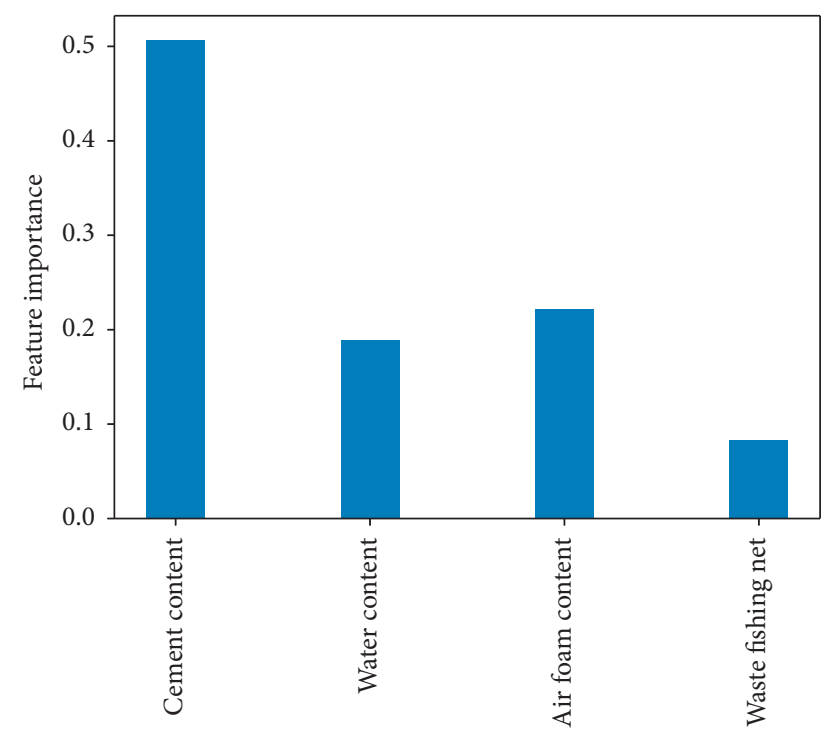

Figure 7: Importance of each input effect on unconfined compressive strength.

layers that contain 12 and 10 neurons, respectively. The performance of the new ANN model is considered to be Pearson's correlation coefficient $R$, Mean Absolute Error (MAE), and Root Mean Square Error (RMSE). Pearson's correlation coefficient $R$ of the model is equal to 0.95 and 0.94 for training and testing parts, respectively. Comparing the performance of the ANN model, Pearson's correlation coefficient $R$ of this paper is slightly lower than that proposed by Park and Kim [7]. However, the prediction of model is successful with lower water content and the large error focus on the high water content of dredged sediment. Overall, it is shown that the performance of the ANN model using two hidden layers seems obviously no greater than that using a single hidden layer.

Figure 7 shows the dependence of unconfined compressive strength $P_{u}$ for each input. The most important input is the cement content used for stabilizing sediments. The least important input is the waste fishing net content. The influence of air foam content on unconfined compressive strength is more important than that of water content.

\section{Conclusion}

In this paper, the ability of artificial intelligence (AI) techniques to predict the compressive strength of stabilized dredged sediments was tested. The dataset used for simulation is collected from experimental results that have been published in literature. To save time and money for conducting experiments, an ANN model was developed. In addition, to confirm and verify the performance of the ANN model, an artificial neural network (ANN) was created and adjusted by back-propagation algorithm (BP) with Levenberg-Marquardt (LMA) algorithm. The compressive strength of stabilized dredged sediments has been predicted by ANN models with network structure [4-12]. The results show that the ANN model can accurately predict the compressive strength of stabilized dredged sediments with low water content. Therefore, this algorithm is a good approach that can be applied for mix design for stabilization/ solidification of dredged sediments. The important input affecting the unconfined compressive strength can be in the following order: cement content $>$ air foam content $>$ water content $>$ waste fishing net. It seems to be very interesting to perform a comparison of performance between the actual model and the new model including normalized inputs, other activation functions, and other learning algorithms in future research. 


\section{Data Availability}

The data supporting this manuscript are from previously reported studies and datasets, which have been cited. The processed data are available from the corresponding author upon request.

\section{Conflicts of Interest}

The author declares that there are no conflicts of interest.

\section{References}

[1] K. Loest and C. M. Wilk, "Brownfield reuse of dredged New York harbor sediment by cement-based solidification/ stabilization," 1998, https://www.osti.gov/biblio/679402brownfield-reuse-dredged-new-york-harbor-sediment-cementbased-solidification-stabilization.

[2] D. Dermatas and X. Meng, "Utilization of fly ash for stabilization/solidification of heavy metal contaminated soils," Engineering Geology, vol. 70, no. 3-4, pp. 377-394, 2003.

[3] C. D. Dunn and J. A Napier, "An evaluation of factors affecting the in vitro bioassay for erythropoietin," Experimental Hematology: Stabilization-Solidification of High Water Content Dredged Sediments, vol. 3, no. 6, p. 362, 1975.

[4] A. Reza, S. Anzum, R. C. Saha, S. Chakraborty, and M. H. Rahman, "Implementation of solidification/stabilization process to reduce hazardous impurities and stabilize soil matrices," E3S Web of Conferences, vol. 96, p. 01003, 2019.

[5] B. T. Vu, V. Q. Tran, Q. D. Nguyen et al., "A geochemical model for analyzing the mechanism of stabilized soil incorporating natural pozzolan, cement and lime," in Proceedings of China-Europe Conference on Geotechnical Engineering, pp. 852-857, Cham, Switzerland, 2018.

[6] T. Tsuchida, "Super geo-material project in coastal zone, development of high quality geo-material by recycling bulky urban waste," in Proceedings of the International Symposium on Coastal Ocean Space Utilization, pp. 391-401, New York, NY, USA, May 1995.

[7] H. I. Park and Y. T. Kim, "Prediction of strength of reinforced lightweight soil using an artificial neural network," Engineering Computations, vol. 28, no. 5, pp. 600-615, 2011.

[8] N. T. B. Phuong, V. P. D. Tri, N. B. Duy, and N. C. Nghiem, "Remote sensing for monitoring surface water quality in the Vietnamese Mekong Delta: the application for estimating chemical oxygen demand in river reaches in binh dai, ben tre," Vietnam Journal of Earth Sciences, vol. 39, no. 3, 2017.

[9] T. Sivasankar, J. M. Lone, K. K. Sarma, and A. Qadir, "Estimation of above ground biomass using support vector machines and ALOS/PALSAR data," Vietnam Journal of Earth Sciences, vol. 41, no. 2, pp. 95-104, 2019.

[10] D. N., S. Trinh, H.-B. Ly, and B. Pham, "Prediction of compressive strength of geopolymer concrete using entirely steel slag aggregates: novel hybrid artificial intelligence approaches," Applied Sciences, vol. 9, no. 6, p. 1113, 2019.

[11] H.-B. Ly, T.-T. Le, L. M. Le et al., "Development of hybrid machine learning models for predicting the critical buckling load of I-shaped cellular beams," Applied Sciences, vol. 9, no. 24, p. 5458, 2019.

[12] L. M. Le, H.-B. Ly, B. T. Pham et al., "Hybrid artificial intelligence approaches for predicting buckling damage of steel columns under axial compression," Materials, vol. 12, no. 10, p. $1670,2019$.
[13] H.-B. Ly, L. M. Le, L. V. Phi et al., "Development of an AI model to measure traffic air pollution from multisensor and weather data," Sensors, vol. 19, no. 22, p. 4941, 2019.

[14] H. Q. Nguyen, H.-B. Ly, V. Q. Tran, T.-A. Nguyen, T.-T. Le, and B. T. Pham, "Optimization of artificial intelligence system by evolutionary algorithm for prediction of axial capacity of rectangular concrete filled steel tubes under compression," Materials, vol. 13, no. 5, p. 1205, 2020.

[15] Q. H. Nguyen, H.-B. Ly, T.-T. Le et al., "Parametric investigation of particle swarm optimization to improve the performance of the adaptive neuro-fuzzy inference system in determining the buckling capacity of circular opening steel beams," Materials, vol. 13, no. 10, p. 2210, 2020.

[16] B. T. Pham, M. D. Nguyen, H.-B. Ly et al., "Development of artificial neural networks for prediction of compression coefficient of soft soil," in CIGOS 2019, pp. 1167-1172, Innovation for Sustainable Infrastructure, Singapore, 2020.

[17] B. T. Pham, T. Nguyen-Thoi, H.-B. Ly et al., "Extreme learning machine based prediction of soil shear strength: a sensitivity analysis using Monte Carlo simulations and feature backward elimination," Sustainability, vol. 12, no. 6, p. 2339, 2020.

[18] O. Moselhi, T. Hegazy, and P. Fazio, "Neural networks as tools in construction," Journal of Construction Engineering and Management, vol. 117, no. 4, pp. 606-625, 1991.

[19] B. Karlik and A. V. Olgac, "Performance analysis of various activation functions in generalized MLP architectures of neural networks," International Journal of Artificial Intelligence and Expert Systems, vol. 1, no. 4, pp. 111-122, 2011.

[20] U. Seiffert, "Training of large-scale feed-forward neural networks," in Proceedings of the 2006 IEEE International Joint Conference on Neural Network Proceedings, pp. 5324-5329, Vancouver, BC, USA, July 2006.

[21] R. ZAYANI, R. Bouallegue, and D. Roviras, LevenbergMarquardt Learning Neural Network for Adaptive Predistortion for Time-Varying HPA with Memory in OFDM Systems, Lausanne, Switzerland, 2008.

[22] S. Trenn, "Multilayer perceptrons: approximation order and necessary number of hidden units," IEEE Transactions on Neural Networks, vol. 19, no. 5, pp. 836-844, 2008.

[23] M. Ibnu Choldun, J. Santoso, and K. Surendro, "Determining the number of hidden layers in neural network by using principal component analysis," in Intelligent Systems and Applicationspp. 490-500, Cham, Switzerland, 2020.

[24] A. M. Neville, Properties of Concrete, Pearson Education, London, UK, 2013.

[25] Hush, "Classification with neural networks: a performance analysis," in Proceedings of the IEEE 1989 International Conference on Systems Engineering, pp. 277-280, New York, NY, USA, August 1989.

[26] H.-B. Ly, L. M. Le, H. T. Duong et al., "Hybrid artificial intelligence approaches for predicting critical buckling load of structural members under compression considering the influence of initial geometric imperfections," Applied Sciences, vol. 9, no. 11, p. 2258, 2019. 